\title{
Neurological Complications Following Frozen Elephant Trunk for Aortic Dissection: What's Truly to Blame?
}

\author{
Sven Zhen Cian Patrick Tan ${ }^{1}$, Hazem Elsantawy ${ }^{2}$, and Amr Abdelhaliem ${ }^{3}$ \\ ${ }^{1}$ Queen Mary University of London Barts and The London School of Medicine and \\ Dentistry \\ ${ }^{2}$ Royal Blackburn Hospital \\ ${ }^{3}$ East Lancashire Hospitals NHS Trust
}

May 24, 2021

\begin{abstract}
Total arch repair (TAR) has become a mainstay of the surgical management of complex pathologies of the ascending aorta and aortic arch, in particular acute Type A aortic dissections (ATAAD). TAR with devices such as the frozen elephant trunk (FET) have been shown to dramatically improve clinical outcomes in such cases. However, TAR with FET remains an immensely challenging procedure, and the risk of debilitating postoperative complications remains high. Spinal cord ischaemia (SCI) and stroke are two particularly tragic adverse outcomes of TAR with FET; it is unsurprising therefore that much research has been done to determine both the underlying cause thereof, and strategies to mitigate this risk. Mousavizadeh and colleagues produced a fascinating systematic review and meta-analysis investigating the relationship between the duration of hypothermic circulatory arrest (HCA) and the risk of developing complications including SCI and stroke. Their data seem to suggest HCA duration is a key factor in causing SCI and stroke following TAR with FET for ATAAD. However, other factors such as stent sizing and landing zone also contribute. Further prospective research into this relationship is recommended to fully elucidate what truly is to blame for these postoperative neurological complications.
\end{abstract}

Neurological Complications Following Frozen Elephant Trunk for Aortic Dissection: What's Truly to Blame?

Running Title: Neurological Complications from Frozen Elephant Trunk

Sven Zhen Cian Patrick Tan ${ }^{1} \mid$ Hazem El Santawy ${ }^{2}$ Amr Abdelhaliem²

1: Barts and The London School of Medicine and Dentistry, Queen Mary University of London, E1 2AD, UK

2: Department of Vascular and Endovascular Surgery, Royal Blackburn Teaching Hospital, Haslingden Road, BB2 3HH, UK

\section{Correspondence:}

Amr Abdelhaliem

Department of Vascular and Endovascular Surgery, Royal Blackburn Teaching Hospital, Haslingden Road, BB2 3HH, UK

Email: amrhaliem@gmail.com

Funding declarations: None to be declared

Conflicts of interest: None to be declared 
Keywords: Aortic arch, arch repair, aortic dissection, frozen elephant trunk, spinal cord ischaemia, stroke

\begin{abstract}
Total arch repair (TAR) has become a mainstay of the surgical management of complex pathologies of the ascending aorta and aortic arch, in particular acute Type A aortic dissections (ATAAD). TAR with devices such as the frozen elephant trunk (FET) have been shown to dramatically improve clinical outcomes in such cases. However, TAR with FET remains an immensely challenging procedure, and the risk of debilitating postoperative complications remains high. Spinal cord ischaemia (SCI) and stroke are two particularly tragic adverse outcomes of TAR with FET; it is unsurprising therefore that much research has been done to determine both the underlying cause thereof, and strategies to mitigate this risk. Mousavizadeh and colleagues produced a fascinating systematic review and meta-analysis investigating the relationship between the duration of hypothermic circulatory arrest (HCA) and the risk of developing complications including SCI and stroke. Their data seem to suggest HCA duration is a key factor in causing SCI and stroke following TAR with FET for ATAAD. However, other factors such as stent sizing and landing zone also contribute. Further prospective research into this relationship is recommended to fully elucidate what truly is to blame for these postoperative neurological complications.
\end{abstract}

\title{
MAIN TEXT
}

We read with great interest the manuscript by Mousavizadeh and Daliri titled "Hypothermic Circulatory Arrest Time affects neurological outcomes of Frozen Elephant Trunk for Acute Type A Aortic Dissection: a systematic review and meta-analysis". ${ }^{1}$ The authors explained with profound clarity the emergence of, and advancements associated with, the frozen elephant trunk (FET) prosthesis as a method of surgical management for complex pathologies of the proximal aorta. In particular, the use of total arch repair (TAR) with FET for rectifying acute type A aortic dissection (ATAAD). The authors highlighted that although the incidence of adverse outcomes following this procedure vary from centre to centre, postoperative complications such as spinal cord ischaemia (SCI), stroke, and in-hospital mortality remain omnipresent risks that could possibly be associated with time spent under hypothermic circulatory arrest (HCA). ${ }^{1}$ Building on this, Mousavizadeh and colleagues undertook a systematic review and meta-analysis to evaluate the association between pre- and intraoperative patient characteristics and post-operative complications, with a view of elucidating any relationship between HCA time and neurological outcome.

Surgeons tasked with repairing ATAAD can elect, depending on myriad preoperative and intraoperative factors, to proceed conservatively or aggressively; that is, whether to execute a hemiarch (HAR) or total arch repair (TAR). ${ }^{2}$ Whereas HAR is typically carried out prior to addressing the many downstream sequelae of ATAAD, the more aggressive TAR often involves deployment of an FET prothesis to enable control of intimal tears and encourage false lumen (FL) remodelling in a single procedure. ${ }^{2}$ Though the TAR approach with FET is purportedly associated with improved FL thrombosis and higher rates of 5-year freedom from rupture, reintervention, and death than HAR, it remains technically demanding and is associated with especially debilitating postoperative complications. ${ }^{2}$ The risk of causing spinal cord ischaemia (SCI), stroke, acute kidney injury, or in-hospital death are particularly pertinent - these are well-documented, common complications associated with arch repair. ${ }^{3,}{ }^{4}$ It is therefore prudent that Mousavizadeh and colleagues have opted to carry out a meta-analysis and systematic review into this relationship.

The investigation benefits from robust and appropriate inclusion and exclusion criteria, which are clearly stated in the manuscript. Notably, by choosing to exclude studies describing non-TAR, non-ATAAD cases, as well as those that do not provide individualised pre- and post-operative data on ATAAD/TAR patients, the authors ensured a high degree of focus and specificity, while still managing to capture a relatively large sample size (3,211 patients across 35 studies). ${ }^{1}$ The investigation also benefits from the execution of suitable and reliable analytic tests. The results obtained via univariate meta-regression corroborate the theory that the risk of SCI $(\mathrm{p}=0.05)$ and postoperative stroke $(\mathrm{p}=0.04)$ is associated with increased HCA time. ${ }^{1}$ Further, the investigators also highlighted pooled estimates for incidence of in-hospital mortality, acute kidney injury, postoperative bleeding, stroke, and SCI that seem to suggest an association between prolonged 
HCA duration and increased risk of postoperative complications. ${ }^{1}$ The authors highlight that there exist discrepancies between their data and that which is already reported, and indeed this is likely due to differences in the overall cohort: Mousavizadeh and colleagues' meta-analysis was carried out on a notably homogenous cohort of only patients with ATAAD undergoing TAR with FET. ${ }^{1}$ Yet, it should be highlighted (as in the manuscript), that the data analysed was subject to significant publication bias. This is apparent in both subjective visual analysis of the Funnel plots and objective analysis via Egger's test used for each outcome. ${ }^{1}$ Further, the Higgins $I^{2}$ values reported for each outcome suggest high degrees of heterogeneity across the 35 studies included - these values ranged between $63.93 \%$ (postoperative stroke) and $86.65 \%$ (AKI). ${ }^{1,5} \mathrm{An}$ exception is post-operative SCI: $I^{2}$ is reported as $19.56 \%$ suggesting relative homogeneity across the studies included. ${ }^{1}$ Finally, the authors note that all studies included were retrospective and reported findings based on non-randomised observations, hence considerable selection bias is therefore implicated. ${ }^{1}$ Yet, it should be highlighted that carrying out prospective, randomised trials are particularly challenging, impractical, and possibly unethical in the field of cardiac surgery. Moreover, as emphasised in the manuscript, there lacks a standard reporting method for TAR with FET, hence a degree of heterogeneity across the 35 studies is inevitable. $^{1,5}$

Choudhury et al. estimate that SCI occurs in up to $7.3 \%$ of patients undergoing FET implantation for ATAAD, and a meta-analysis by Rezaei et al. reported a pooled estimate of stroke and paraplegia occurring in $7 \%$ and $3.5 \%$ of cases respectively. ${ }^{6,7}$ Prolonged cardiopulmonary bypass (CPB), HCA, and anterograde cerebral perfusion durations are well-documented determinants of adverse postoperative events, neurological damage included. ${ }^{8}$ Jiang et al. suggest that executing TAR with FET at moderate (rather than deep) hypothermia $\left(28^{\circ} \mathrm{C}\right)$ can help reduce overall HCA duration, and attenuate the risk of neurological injury. ${ }^{8}$ Selective perfusion via the left subclavian artery (LSA) has also been proposed as a means of improving spinal cord perfusion via collateral vasculature during HCA, thereby further lowering the risks of HCA-associated SCI and stroke. ${ }^{9}$ Furthermore, the distal landing zone and graft length (longer than $15 \mathrm{~cm}$, extending beyond T8) of the FET have previously been identified as a potential risk factor for postoperative SCI; indeed, Jiang et al.suggest that decreased occlusion of the intercostal vessels, achieved via proximalisation of FET stent to Zone 0, may contribute do decreased SCI risk.8, 9, 10

10,11

To conclude, the findings in this manuscript do seem to point towards HCA duration as one of the key contributing factors towards causing postoperative neurological complications, such as SCI and stroke. ${ }^{1}$ The investigation was carried out with robust methodology and analytic approaches, and all potential limitations thereof are reported by the authors. ${ }^{1}$ This meta-analysis and systematic review therefore provides valuable insight into the relationship between HCA duration and postoperative neurological complications, and recommends further prospective research into the area to fully ascertain whether HCA duration is the true culprit in causing SCI and stroke.

\section{REFERENCES}

Mousavizadeh M, Daliri M, Aljadayel HA, Idhrees M, Rezaei Y, Bashir M, Hosseini S. Hypothermic Circulatory Arrest Tin

1. Bashir M, Harky A. Current status in decision making to treat acute type A dissection: limited versus extended repair. The Journal of cardiovascular surgery. 2020 Jan 23.

2. Jakob H, Dohle D, Benedik J, Jánosi RA, Schlosser T, Wendt D, Thielmann M, Erbel R, Tsagakis K. Long-term experience with the E-vita Open hybrid graft in complex thoracic aortic disease. European Journal of Cardio-Thoracic Surgery. 2017 Feb 1;51(2):329-38.

3. Beckmann E, Martens A, Korte W, Kaufeld T, Krueger H, Haverich A, Shrestha M. Open total arch replacement with trifurcated graft and frozen elephant trunk. Annals of cardiothoracic surgery. 2020 May;9(3):170.

4. Lin L, Chu H. Quantifying publication bias in meta-analysis. Biometrics. 2018 Sep;74(3):785-94.

5. Choudhury RY, Basharat K, Zahra SA, Tran T, Rimmer L, Harky A, Idhrees M, Bashir M. "Prox- 
imalization is Advancement"-Zone 3 Frozen Elephant Trunk vs Zone 2 Frozen Elephant Trunk: A Literature Review. Vascular and Endovascular Surgery. 2021 Mar 23:15385744211002493.

6. Rezaei Y, Bashir M, Mousavizadeh M, Daliri M, Aljadayel HA, Mohammed I, Hosseini S. Frozen elephant trunk in total arch replacement: A systematic review and meta-analysis of outcomes and aortic proximalization. Journal of Cardiac Surgery. 2021 Mar 4.

7. Jiang SM, Ali Hassan SM, Nguyen G, Bisleri G. Zone 0 frozen elephant trunk for type A retrograde acute aortic dissection following endovascular stenting of the arch. Journal of Cardiac Surgery. 2021 Feb 22.

8. Leontyev S, Tsagakis K, Pacini D, Di Bartolomeo R, Mohr FW, Weiss G, Grabenwoeger M, Mascaro JG, Iafrancesco M, Franke UF, Gobel N. Impact of clinical factors and surgical techniques on early outcome of patients treated with frozen elephant trunk technique by using EVITA open stent-graft: results of a multicentre study.

9. Preventza O, Liao JL, Olive JK, et al. Neurologic complications after the frozen elephant trunk procedure: a meta-analysis of more than 3000 patients. In: J Thorac Cardiovasc Surg. 2020;160(1): 20-33. e4. 